\title{
Discussion Regarding the Termination of Pregnancy Due to Cancer Diagnosis
}

National Cancer Institute

\section{Source}

National Cancer Institute. Discussion Regarding the Termination of Pregnancy Due to

Cancer Diagnosis. NCI Thesaurus. Code C137831.

Discussions between a physician and a pregnant cancer patient regarding the effects of anticancer drugs on the developing fetus or the possibility of long-term complications after the exposure to drugs and radiation, as well as complications to treatment, with the goal toward deciding if the pregnany should be terminated. 